\title{
CALCULATION OF NODAL PRICES FOR HEAT ENERGY IN HEAT SUPPLY SYSTEMS
}

\author{
Andrey Penkovskii ${ }^{1}$,Oleg Khamisov ${ }^{1}$, Angelica Kravets $^{1^{*}}$ \\ ${ }^{1}$ Melentiev Energy Systems Institute of Siberian Branch of the Russian Academy of Sciences, Lermontov str., 130, Irkutsr, 664033, \\ Russia
}

\begin{abstract}
The article is devoted to the calculation of nodal prices for heat energy in heat supply systems. The problem, mathematical model and method of calculating nodal prices of heat energy for all consumers of the heat supply system considered, taking into account the different cost of heat production by sources, optimal flow distribution, and placement of consumers in the heat network (distance from the source). As the main computational tool for calculating nodal prices for heat energy, the Lagrange multiplier method used in the problem of modes optimizing in heat supply system, which allows explaining in detail the formation of the price for heat energy in each node. With the help of the proposed methodological support, practical research has been carried out for real heat supply system.
\end{abstract}

\section{Introduction}

Structural transformations in the heat power industrial of Russia, associated with the processes of liberalization in the energy sector in the 90 s of the twentieth century, led to new economic relations among producers and consumers of heat energy and the creation of a heating market. At the same time, there was virtually no legal framework that would regulate relations arising in connection with the production, transportation and consumption of heat energy.

Only 20 years after the collapse of the USSR was the main Russian Federation Federal law of 27.07.2010 No.190-FL law "On heat supply" [1] adopted (with the exception of certain regulations). Its adoption was associated with fundamental changes in the political, socio-economic life of the country, directly affecting the solution of such an important problem as ensuring reliable and uninterrupted supply of heat energy to consumers in the territory of the Russian Federation and creating a unified model for organizing the heat energy market.

The new model of district heating market provides for the unification of all functions of the heat supply system (HSS) including the production, transportation and sale of energy in the framework of the Unified Heat Supply Organization (UHSE). The merger of the main assets and processes of heat supply management forms the structure of UHSO as the only seller in the district heating market in the form of a natural monopolist.

According to current legislation, district heating markets in Russia are regulated natural monopolies, where the price of heat energy is calculated based on the actual total costs incurred for the production and transportation of heat energy. At the same time, consumers pay for heat energy at an average tariff. However, this approach does not take into account the fact that all heat consumers are in different conditions and the real cost of heat supplied is not the same. This approach does not reflect the emerging market relations among producers (suppliers) and consumers of heat energy.

To assess the effectiveness of the functioning and development of HSS in the market conditions, a transition to nodal (differentiated) calculation of prices for heat energy is required, which will allow taking into account the real costs of covering the heat load of each specific consumer. This approach (nodal price calculation) is well established in the power industry [2$6]$. The calculation of nodal prices is based on modes optimization of the established modes of electric power systems based on the criterion of the minimum total cost of electricity production. Electricity prices are determined for each node of the electric power system as dual estimates or Lagrange multipliers to the active power balance equations at the nodes.

In HSS, the calculation of nodal prices [7-9] is based on the calculation of heat-hydraulic modes in HSS, taking into account the different cost of heat production by sources, the actual flow distribution in heat networks, the location of consumers in the heat network (distance from heat sources), its structure and parameters. The disadvantage of this approach is the lack of consideration of the market (margin) specifics of pricing for heat energy.

In this article, we propose to use an approach for calculating nodal prices for heat energy based on the modes optimization in HSS with its further reduction to the optimality conditions based on the Lagrange function, while the indeterminate Lagrange multipliers obtained in the calculation process will be interpreted as nodal (marginal) prices for heat energy.

\section{Calculation of nodal prices for heat energy in HSS}

In mathematical modeling of a HSS, which is organized in the format of a UHSO and has both heat sources (HS) and heat networks (HN), we assume that it has a nodal structure and is modeled by a hydraulic

\footnotetext{
* Corresponding author: kravets@isem.irk.ru
} 
circuit (HC) consisting of $m$ nodes and $n$ branches [10]. This HC structure described by a complete connection matrix $\overline{\mathrm{A}}$ in which the number of rows matches the number of nodes, and the number of columns matches the number of branches.

Elements of the $a_{i j}$ matrix $\overline{\mathrm{A}}$ are defined by the following conditions:

$$
\begin{aligned}
& 0, \text { if the branch } \mathrm{i} \text { has no } \\
& \text { connection to node } \mathrm{j} ; \\
& a_{i j}= 1, \text { if the flow on the } \mathrm{i} \text { branch comes } \\
& \text { from node } \mathrm{j} \\
&-1, \text { if the thread on the branch } \\
& \mathrm{i} \text { enters the node } \mathrm{j}
\end{aligned}
$$

Let us denote the set of nodes by $J=\{j: j=1, \ldots, m\}$ and the set of branches by $I=\{i: i=1, \ldots, n\}$. The following notations are also used: $J_{\mathrm{HS}} \quad J$ is a set of heat sources, $J_{\mathrm{CON}} \quad J$ is a set of consumers, $J_{0} \quad J$ is a set of branching nodes without producers and consumers.

The calculation of nodal prices for heat energy is based on the use of optimality conditions for the problem of modes optimizing in HSS, and above all its dual variables (indeterminate Lagrange multipliers) [11, 12]. From [10] it is known that the use of optimality conditions based on the method of indeterminate Lagrange multipliers to solve the problem of finding the optimal modes of HSS on energy criterion (minimum of the total losses to overcome friction in HSS compliance with the material balance at the nodes and end ratio) allows to determine the values of the variables of the objective function and optimal values of the Lagrange multipliers, which are interpreted as nodal pressure in the HSS. Thus, to jump to the definition of nodal prices for heat energy, it is enough to formulate the problem of finding the optimal modes in HSS on the economic criterion, as it is formulated, for example, the power industry [2-6], namely search minimum total cost of production and transportation of heat energy in HSS:

$$
\begin{gathered}
Z^{\mathrm{TOTAL}}={ }_{j J_{\mathrm{HS}} \quad} Z_{j}^{\mathrm{HS}}\left(Q_{j}^{\mathrm{HS}}\right)+Z^{\mathrm{HN}}{ }_{(\mathbb{R})} \mathrm{min}, \\
\mathbf{A x}=\mathbf{Q}, \\
\mathbf{h}=\mathbf{S X x},
\end{gathered}
$$

where $-Z^{\text {TOTAL }}$ total costs of production and transportation of heat energy to consumers, EUR; $Z_{j}^{\mathrm{HS}}\left(Q_{j}^{\mathrm{HS}}\right)-$ is heat production costs of the $j$-th heat source, EUR; $Z^{\mathrm{HIN}}$ - is heat transportation cost, EUR.; $\mathrm{A}$ - the matrix of incidence $(\mathrm{m}-1)$ linearly independent nodes and $\mathrm{n}$ branches; $\mathrm{x}=\left(x_{1}, \ldots, x_{n}\right)^{\mathrm{T}}$ - vector of flow rates, $\mathrm{t} / \mathrm{h} ; \mathbf{Q}=\left(Q_{1}, \ldots, Q_{m-1}\right)^{\mathrm{T}}-$ vector nodal sources or sinks, the $\mathrm{t} / \mathrm{h} ; \quad \mathbf{h}=\left(h_{1}, \ldots, h_{n}\right)^{\mathrm{T}}$ - vector of pressure losses on branch, Pa.; $\mathrm{S}=\operatorname{diag}\left(s_{1}, \ldots, s_{n}\right)$,
$\mathbf{X}=\operatorname{diag}\left(\left|x_{1}\right|, \ldots,\left|x_{n}\right|\right)$ - diagonal matrices of hydraulic resistances and flow rates, respectively

Production costs of heat sources can be represented as a functional relationship among the costs and heat produced by the heat sources, that includes variable and fixed components. It is difficult to derive the cost functions since each type of the production equipment has characteristics described by separate expressions.

Information on these characteristics is taken from normative documents of the base equipment. After testing, the characteristics are corrected taking into account real values of various internal and external factors such as vapor pressure in boiler, type and cost of used fuel, outdoor temperature and so on. Our experience shows that the best approximation of the correspondence between costs and volumes of produced heat is given by the function [13], EUR.:

$$
\left.Z_{j}^{\mathrm{HS}}\left(Q_{j}^{\mathrm{HS}}\right)=\alpha_{j} \times Q_{j}^{\mathrm{HS}}\right)^{2}+\beta_{j} \chi_{j}^{\mathrm{HS}}+\gamma_{j},
$$

where $-\alpha_{j},(\mathrm{EUR} /(\mathrm{GJ} / \mathrm{h}))^{2}, \beta_{j}, \mathrm{EUR} /(\mathrm{GJ} / \mathrm{h}) ; \gamma_{j}$, (EUR) - are approximation coefficients of a heat source cost characteristic.

Since the coefficients $\alpha_{j}, \beta_{j}$ and $\gamma_{j}$ are positive, the cost function is a strongly convex increasing positive function at $Q_{j}^{\mathrm{HS}} \quad 0$.

The transportation cost of heat energy is determined by the following dependence [14]:

$$
Z^{\mathrm{HN}}={ }_{i=1}^{n} Z_{i}^{\mathrm{INN}}\left(x_{i}\right)=F_{1}+F_{2} \times_{i=1}^{n} x_{i} \varkappa_{i} \text {, }
$$

where $F_{1}=f \times\left[a_{i}+b_{i} \Varangle_{i}^{0.19 u_{i}} \times_{i}^{-0.19 \varkappa_{i}} \Varangle_{i}^{0.19 u_{i}}\right] \aleph_{i}$ $i=1$

-semi-fixed costs of heat network, EUR.; $f=0.075-$ the share of conditionally constant maintenance (on the maintenance) costs for the heating network; $a_{i}$ (EUR $/ \mathrm{m}), \quad b_{i}\left(\mathrm{EUR} / \mathrm{m}^{u_{i}+1}\right), \quad u_{i}$ (dimensionless value) are approximation coefficients of numerical values for unit cost of laying pipelines of different diameters; $\chi_{i}{ }^{-}$ coefficient dependent on roughness of conduit (dimensionless value); $l_{i}$ - length of the $i$-th network section, $\mathrm{m} ; \quad F_{2}=\frac{C_{e}}{367.2 \times}-$ is coefficient at semivariable costs of heat network; $C_{e}-$ is a unit cost of electricity, EUR $/ \mathrm{kWh} ; \quad-$ is pumping station efficiency, $\%$.

Problem (2)-(4) is a description of the steady-state mode in the HSS, taking into account the optimal flow distribution of the heat follows in the heat networks and the optimal volumes of heat energy production by sources. Since expression (4) is a monotonically 
increasing function that has the properties of odd and smoothness [10], to reduce the dimension of the problem (2)-(4), you can replace the variables by substituting expression (4) in the cost function in heat networks. Thus, the problem of modes optimizing in HSS will be written as follows:

$$
\begin{gathered}
Z^{\text {TOTAL }}={ }_{j} J_{\mathrm{HS}}\left[\alpha_{j} \times\left(Q_{j}^{\mathrm{HS}}\right)^{2}+\beta_{j} \times Q_{j}^{\mathrm{HS}}+\right. \\
\left.+\gamma_{j}\right]+F_{1}+F_{2} \times_{i=1}^{n} x_{i}^{2} \Varangle x_{i} \mid \times_{i} \text { @ min }
\end{gathered}
$$

\section{Method for solving the problem of modes optimizing in HSS}

The formulated problem (7) - (8) of modes optimization in HSS due to the convexity of the cost functions of the heat sources and the flow rates on sections of the heat network has a unique global solution [10]. The constraints in the problem under study are linear, therefore, regular [11], therefore, to write down the necessary optimality conditions, we use the Lagrange function in the following form:

$$
\begin{aligned}
& L(\mathrm{Q}, \mathrm{x}, \lambda)={ }_{j} J_{\mathrm{HS}}\left[\alpha_{j} \rtimes Q_{j}^{\mathrm{HS}}\right)^{2}+\beta_{j} \chi_{j}^{\mathrm{HS}}+ \\
& \left.+\gamma_{j}\right]+F_{1}+F_{2} \times_{i=1}^{n} x_{i}^{2} x_{i} \mid x_{i}+\lambda \times(\mathbf{A x}-\mathbf{Q}),
\end{aligned}
$$

where $\lambda=(1, \ldots, m)^{\mathrm{T}}-$ the vector of the Lagrange multipliers.

The necessary optimality conditions in this case are sufficient and represent a system of partial differential equations of the Lagrange function (9):

$$
\begin{aligned}
& \frac{L(\mathrm{Q}, \mathrm{x}, \lambda)}{\pi Q_{j}^{\mathrm{HS}}}=2 \succ_{j} \succ_{j}^{\mathrm{HS}} \rtimes_{j}-j=0, j \quad J_{\mathrm{HS}} \\
& \frac{\prod L(\mathrm{Q}, \mathrm{x}, \lambda)}{\rrbracket x_{i}}=F_{2} \nVdash x_{i}^{2} \rtimes \operatorname{sign}\left(x_{i}\right) x_{i}+ \\
& \left.2 x_{i} \ngtr_{x_{i}} \mid \rtimes_{i}\right]+{ }_{j} a_{j i} \times_{j}=0, i \quad I \\
& \frac{\prod L(\mathrm{Q}, \mathrm{x}, \lambda)}{\mathbb{T} \lambda}=\mathrm{Ax}-\mathrm{Q}=0 .
\end{aligned}
$$

The solution of the system of equations (10) allows to define variables of function of the total cost function of HSS, in which the value of the Lagrange function , and the value of the objective function the total costs of HSS ( $Z^{\text {TOTAL }}$ ) correspond to the extremum. Also, in the course of solving the system of equations (10), dual variables to constraints are determined. The vector of indeterminate Lagrange multipliers $\lambda=(1, \cdots, m)^{\mathrm{T}}$ to the restrictions (8) on the material balance in the nodes of the HSS interpreted from an economic point of view as the nodal equilibrium prices for heat energy in the HSS, including the equilibrium prices of heat energy production by heat sources and the equilibrium prices of all consumers.

According to the calculated nodal prices for heat energy for consumers and the obtained optimal value of the total costs in the HSS, it is possible to determine the amount of profit that the UHSO will receive when supplying consumers with heat energy, EUR:

$$
P={ }_{j J_{\mathrm{HS}}} \succ_{j}-Z^{\text {TOTAL }}
$$

In order to identify the most efficient and inefficient zones in the HSS using nodal prices for heat energy for consumers, it is proposed to introduce a single price for heat energy for all consumers in the form of a weighted average price, i.e., which will take into account both the volume of heat energy consumption of an each consumer, and the price for its consumption, EUR/GJ:

$$
w=\frac{j J_{\mathrm{HS}} j^{\chi_{j}}}{j J_{\mathrm{HS}} Q_{j}} .
$$

Thus, the algorithm for calculating the nodal prices of production and consumption of heat energy and obtaining technical and economic indicators of HSS, including the costs of production and transportation of heat energy, as well as the profit of UHSO from the sale of heat energy to consumers, in the problem of modes optimizing in HSS based on the Lagrange multiplier method is as follows:

Step 1. The design scheme of HSS indicating the locations of heat sources and consumers is formed.

Step 2. The raw data is formed, including the heat loads of consumers, the cost functions of heat sources, the physical and technical parameters of heat network (lengths, diameters, resistances of sections of the heat network, the efficiency of pumping units, etc.).

Step 3. The problem of modes optimization in HHS are formed by the criterion of finding the minimum total costs in the heating system, taking into account the restrictions on material balances in the nodes of the heat network.

Step 4. The Lagrange function compiled.

Step 5. Partial derivatives of the Lagrange function with respect to variables are determined and equated to zero.

Step 6. The system of equations of the obtained partial derivatives is solved.

Step 7. The resulting values of the variables are used to determine the total costs HSS.

Step 8. With the help of the calculated nodal prices for heat energy for the consumer and the total costs in HSS the calculated value of the received profit of the UHSO from the sale of heat energy to consumers according to the formula (11).

Step 9. The weighted average price is calculated according to the formula (12) in order to determine the less and more costly nodes-consumer. 


\section{Case study}

For practical testing of the developed scientific and methodological support for calculating nodal prices for heat energy, consider an example of HSS consisting of 11 branches and 8 nodes, of which 6 nodes are consumers of heat energy and 2 nodes are a heat sources. Figure 1 shows the design schema of the heat supply system.

Fig. 1. Design scheme of the heat supply system

Input data for conclusion nodal prices of HSS:

1. The resistances branches shown in table 1 .

Table 1. The resistance branches

\begin{tabular}{|c|c|c|c|}
\hline Branch & $\begin{array}{c}\text { Resistance, } \\
\mathrm{m} \mathrm{xh}^{2} / \mathrm{t}^{2}\end{array}$ & Branch & $\begin{array}{c}\text { Resistance, } \\
\mathrm{m} \mathrm{\aleph h}^{2} / \mathrm{t}^{2}\end{array}$ \\
\hline 1 & 0.000015 & 7 & 0.000019 \\
\hline 2 & 0.00002 & 8 & 0.000016 \\
\hline 3 & 0.000017 & 9 & 0.000014 \\
\hline 4 & 0.00001 & 10 & 0.000018 \\
\hline 5 & 0.00012 & 11 & 0.000011 \\
\hline 6 & 0.000013 & - & - \\
\hline
\end{tabular}

2. The lengths of branches shown in table 2 .

Table 2. The length of pipeline sections

\begin{tabular}{|c|c|c|c|}
\hline Branch & Length, $\mathrm{m}$ & Branch & Length, $\mathrm{m}$ \\
\hline 1 & 1000 & 7 & 1300 \\
\hline 2 & 1500 & 8 & 1300 \\
\hline 3 & 1200 & 9 & 1400 \\
\hline 4 & 900 & 10 & 1250 \\
\hline 5 & 1100 & 11 & 1100 \\
\hline 6 & 1600 & - & - \\
\hline
\end{tabular}

3. Unit cost of electricity: $C_{e}=0.03 \mathrm{EUR} / \mathrm{kW} \varlimsup_{1}$.

4. Pumping station efficiency: $\eta=0.7$.

5. The share of conditionally constant maintenance costs for the $\mathrm{HN} f=0.075$.

6. The functions of heat production costs of the heat sources in table 3 .

Table 3. The functions of heat production costs

\begin{tabular}{|l|l|}
\hline \multicolumn{2}{|c|}{ The cost function, EUR } \\
\hline HS -1 & $Z\left(Q_{1}\right)=1.15 \times 10^{-4} \times Q_{1}{ }^{2}+1.03 \times Q_{1}+770$ \\
\hline HS-2 & $Z\left(Q_{5}\right)=5.7 \times 0^{-3} \times Q_{5}{ }^{2}+1.12 \times Q_{5}+702$ \\
\hline
\end{tabular}

7. Heat loads of consumers presented in table 4.

Table 4. Heat loads of consumers

\begin{tabular}{|c|c|c|c|}
\hline Node & Load, GJ/h & Node & Load, GJ/h \\
\hline 2 & 838,0 & 6 & 737,4 \\
\hline 3 & 670,4 & 7 & 603,4 \\
\hline
\end{tabular}

\begin{tabular}{|l|c|c|c|}
\hline 4 & 502,8 & 8 & 905,0 \\
\hline
\end{tabular}

8. Numerical values of the coefficients for unit cost of laying pipelines: $a=146, b=1405, u=1,45$.

9. The coefficient depending on the roughness of the pipeline:

$$
\begin{aligned}
& \chi_{1}=\chi_{2}=\chi_{3}=\chi_{4}=\chi_{5}=\chi_{6}=\chi_{7}= \\
& =\chi_{8}=\chi_{9}=\chi_{10}=\chi_{11}=0,01277 .
\end{aligned}
$$

Simulation of the heat supply system shown in fig. 1 was performed in the "GAMS" (General Algebraic Modeling System) computing environment. The main estimated technical and economic indicators of UHSO are presented in table 5 and the nodal prices for consumers in table 6 .

Table 5. The technical and economic indicators of the UHSO

\begin{tabular}{|l|c|}
\hline Indicators & Values \\
\hline Total production volume GJ/h, including: & 4257 \\
\hline HS-1 & $2539(59.6 \%)$ \\
\hline HS-2 & $1718(40 . \% 4)$ \\
\hline $\begin{array}{l}\text { Total cost of heat energy production by } \\
\text { HS EUR, including: }\end{array}$ & 7004 \\
\hline HS -1 & 4135 \\
\hline HS- & 2869 \\
\hline Nodal prices of heat sources EUR/GJ, including.: \\
\hline HS-1 & 28,4 \\
\hline HS-2 & 24,5 \\
\hline Transportation costs, EUR. & 1173 \\
\hline Total costs, EUR. & 8177 \\
\hline Revenue UHSO, EUR. & 9421 \\
\hline Profit, EUR, \%. & $1244(13.2 \%)$ \\
\hline
\end{tabular}

As a result of the solution, the following nodal prices are obtained, shown in table 6.

Table 6. The nodal prices for consumers

\begin{tabular}{|c|c|c|c|c|}
\hline Node & $\begin{array}{c}\text { Nodal price } \\
\text { for heat } \\
\text { energy, } \\
\text { EUR /GJ }\end{array}$ & Node & $\begin{array}{c}\text { Nodal price } \\
\text { for heat } \\
\text { energy, } \\
\text { EUR /GJ }\end{array}$ & $\begin{array}{c}\text { Weighted } \\
\text { average } \\
\text { price, } \\
\text { EUR/GJ }\end{array}$ \\
\hline 2 & 37.95 & 6 & 38.90 & \multirow{2}{*}{38.85} \\
\hline 3 & 40.03 & 7 & 40.28 & \multirow{2}{*}{5} \\
\hline 4 & 38.67 & 8 & 37.90 & \\
\hline
\end{tabular}

Table 1 shows that to cover a given total load of 4 $257 \mathrm{GJ} / \mathrm{h}, \mathrm{HS}-1$ and HS-2 produced $2539 \mathrm{GJ} / \mathrm{h}$ and $1718 \mathrm{GJ} / \mathrm{h}$ heat energy, respectively. At the same time, prices for heat production for HS-1 will amount to 28.4 EUR/GJ and 24.5 EUR/GJ for HS-2. The costs of the UHSO for the production and transportation of heat energy was $8177 \mathrm{EUR}$, and the cost of a unit of heat energy was 33.7 EUR/GJ.

The resulting nodal prices for heat energy consumers are shown in table 2 and in figure 2. 


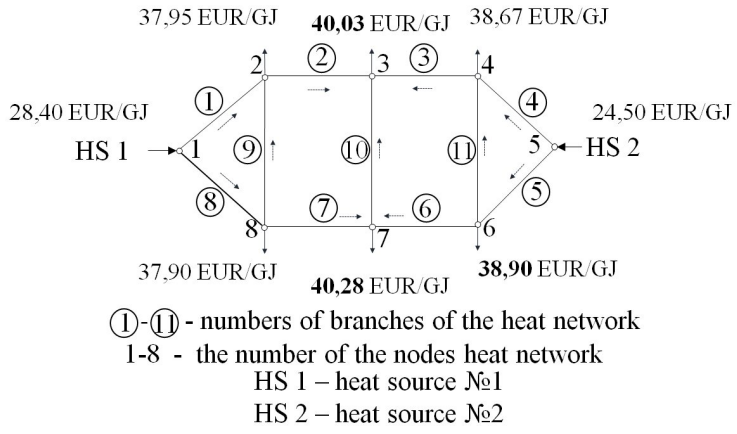

Fig.2. Formation of the price field on the design scheme of the heat supply system.

Figure 2 shows that the nodal prices for heat energy grow in the direction of the established optimal flow distribution in the heat network, and reach maximum values in nodes $3-40.03 \mathrm{EUR} / \mathrm{GJ}$ and $7-40.28 \mathrm{EUR} / \mathrm{GJ}$, which are as far as possible from the heat sources HS-1 and HS-2. If the calculation of prices for heat energy was calculated as a weighted average, it would be 38.85 EUR/GJ for all consumers. Relative to this price, the nodal prices for heat energy in nodes 3,6 , and 7 are higher by $1.18 \mathrm{EUR} / \mathrm{GJ}, 0.05 \mathrm{EUR} / \mathrm{GJ}$, and $1.43 \mathrm{EUR} / \mathrm{GJ}$, respectively. Thus, if the transition from differentiated prices for heat energy to the weighted average price for heat energy for all consumers, then consumers located in nodes 2, 8 and 4 will pay the actual costs of transporting heat energy to consumers located in nodes $3,6,7$. As noted in [8], the transition to differentiated prices can affect many social, organizational, and technological problems, affect the cost of housing and the development of infrastructure in localities near cheap heat sources. At the same time, this approach allows you to perform technical and financial analysis of HSS when planning modes, as well as when evaluating the current mode in HSS.

\section{Conclusions}

In this article, the method for calculating the nodal prices for heat energy in heat supply systems is developed. It is based on the problem of modes optimization in heat supply system according to the criterion of minimum total costs for the production and transportation of heat energy. To find the optimal solution to this problem, an approach based on the Lagrange multiplier method is proposed. It is shown that undefined Lagrange multipliers under balance constraints (Kirchhoff first law) are interpreted as nodal prices. This approach allows us to determine the optimal flow distribution in the heat network, the optimal distribution of loads among heat sources and their corresponding costs, and the nodal prices of heat production and consumption. With the help of the developed methodology, practical studies were performed on the real heat supply system. The nodal prices obtained in the calculations are marginal, i.e. based on the calculation of marginal costs for the production and transportation of an additional unit of heat energy. The proposed approach is implemented in the form of an appropriate algorithm and allows you to model heat supply systems of any scale, capacity and take into account different types of heat sources.

\section{References}

1. Federal Law of the Russian Federation "on heat supply" No. 190-FZ of 27.07.2010.

2. T. A Vaskovskaya, Power plant 1, pp. 25-32 ( 2017)

3. Pan Guangsheng, Gu Wei, Wu Zhi, Lu Yuping, Lu Shuai. Applied Energy 239, pp.280-295 (2019)

4. F. Kunz, K. Neuhoff, J. Rosellón. Energy Economics 6, pp.176-185 (2016)

5. B. G Bulatov., V. O. Ser. Energy. 12, 34 (2009)

6. A. Z.Gamm, I. I. Golub, A.V. Batyunin, A. A. GAMM, Electricity. 10 pp. 17-24. (2005)

7. Z. I. Shalaginova, Teploenergetika 10, pp. 96108 (2018)

8. Z. I. Shalaginova, N. N. Novitsky, V. A. Stennikov, Pipeline energy systems. Methods of mathematical modeling and optimization: Collection of scientific papers. Nauka, Novosibirsk pp. 210-221 (2007)

9. Z. I. Shalaginova, Pipeline energy systems. Development of the theory and methods of mathematical modeling and optimization. (Nauka, pp. Novosibirsk, pp.292-303 2008)

10. A. P.Merenkov, V. Ya Khasilev. Theory of hydraulic circuits( M.: Nauka, Novosibirsk 1985)

11. J. L. Lagrange, Analytical mechanics 1 (TRANS. with French M.-L.: GONTI, 1938)

12. M. Bazara, Shetty K. Theory and algorithms (Mir Moscow (1982)

13. A. V. Penkovskii, V. A. Stennikov, E. E. Mednikova, I. V. Postnikov, Energy. 161. pp. 193-201 (2018)

14. Sennova E. V., Sidler V. G. Mathematical modeling and optimization of developing heat supply systems (Nauka, Novosibirsk 1987) 